\title{
MISC: A Kawasaki-like hyperinflammation syndrome in COVID-19
}

\author{
Imran Raza ${ }^{1}$, Zubia Zaman ${ }^{1}$, and Yamna Waseem ${ }^{1}$ \\ ${ }^{1}$ Dow University of Health Sciences
}

June 9,2020

A surge in pediatric patients presenting with an unfamiliar "multi-system inflammatory state" has been recorded since the coronavirus pandemic. A subgroup of these children tested positive for COVID-19 or had antibodies against SARS-CoV-2 indicating prior infection. [2] Despite patients under 20 years of age who exhibit mild symptoms, comprise no more than two percent of COVID-19 cases, recent fatalities due to a pediatric hyper inflammatory state suggests an unexplored bigger picture. [1,2]

Multi-System Inflammatory Syndrome in Children (MIS-C) have been reported in Europe and North America. New York, being the epicenter of this disorder, recorded over 100 cases with three fatalities. [3] Patients should present with persistent fever, multisystem organ involvement (shock, hypotension), inflammation (neutrophilia, elevated CRP, lymphopenia), and/or COVID-19 positivity or exposure for a definitive diagnosis of MIS-C. Moreover, other microbial causes such as (Staphyloccocal shock) should be excluded. [9] These patients display overlapping symptoms with Toxic Shock Syndrome (TSS) and Kawasaki disease. [2] MIS-C patients do not exhibit typical COVID-19 symptoms like cough or difficulty in breathing as it is considered to be a post infectious state generated by the body's own immune system. [4]

Kawasaki Disease $(\mathrm{KD})$ is an acquired vasculitis of medium-sized arteries that primarily affects children younger than 5 years of age and is a leading cause of acquired cardiac disease in the United States. The hallmark of Kawasaki Disease includes a persistent high fever (over $101^{\circ} \mathrm{F}$ ) for at least 4 days in addition to rash, redness to eyes, lips swelling, pallor in distal limbs and neck swelling. [5] Although MIS-C borrows its signs and symptoms from both KD and TSS, it is considered a separate disease. Kawasaki disease commonly affects children of Asian ancestry, while MIS-C hasn't been reported in Asian countries. [6] Moreover, some MIS-C patients tend to be older than typical KD patients. According to a few case reports, a higher abnormality of biomarkers of inflammation and immune function is found than in Kawasaki Disease patients. [5]

MIS-C shows a spectrum of Kawasaki resembling states which also define the management regimen of the patient. This includes a viral sepsis with shock and hypotension requiring fluid resuscitation and inotropic support which is usually found in older teens and young adults. The second resembles Kawasaki Disease more closely, affecting young children. The latter is treated using intravenous immunoglobulin, corticosteroids and biologics including infliximab and anakinra. $[6,7]$ In case of myocardial involvement (elevated troponin I, ECG fluctuations), the patient should be provided critical care with Enoxaparin (anti-coagulant) prophylaxis and disease modifying agents. Daily lab tests for inflammatory markers including CRP, ESR along with chest X-ray and ECG should be conducted to monitor treatment and adjust dosages accordingly. [8, 9]

Although a rare condition, prompt diagnosis and treatment of MIS-C is critical to decrease both the death toll and current burden on the healthcare system. A procedure developed by Royal College of Pediatrics and Child Health has been developed for the management and treatment of these kids which can be used by health experts worldwide. [9] Increased research regarding this disease would allow for efficient treatment of patients.

\section{References}


1. Hosppeds.aappublications.org. https://hosppeds.aappublications.org/content/hosppeds/early/2020/04/06/hpeds.20200123.full.pdf. Published 2020. Accessed June 9, 2020.

2. Coronavirus: What is Kawasaki disease and its possible link with COVID-19 in children? Msn.com. https://www.msn.com/en-xl/news/other/coronavirus-what-is-kawasaki-disease-andits-possible-link-with-covid-19-in-children/ar-BB13AqKG. Published 2020. Accessed June 9, 2020.

3. South Korea examines first suspected cases syndrome kids linked COVID-19. The Express Tribune. https://tribune.com.pk/story/2229451/3-south-korea-examines-first-suspected-cases-syndromekids-linked-covid-19/. Published 2020. Accessed June 9, 2020.

4. (COVID-19) C. COVID-19 May Trigger Rare Complication In Children. WebMD. https://www.webmd.com/lung/news/20200514/covid19-may-trigger-rare-complication-in-children. Published 2020. Accessed June 9, 2020.

5. COVID-19 and Kawasaki Disease: What Parents Need to Know. Nationwidechildrens.org. https://www.nationwidechildrens.org/family-resources-education/700childrens/2020/05/covid-19and-kawasaki-disease-what-parents-need-to-know. Published 2020. Accessed June 9, 2020.

6. @theresakmach F. What Experts Know About a Rare Inflammatory Syndrome Linked to COVID-19. Smithsonian Magazine. https://www.smithsonianmag.com/smart-news/what-you-should-know-aboutrare-covid-19-inflammation-syndrome-180974995/. Published 2020. Accessed June 9, 2020.

7. Thelancet.com. https://www.thelancet.com/pdfs/journals/lancet/PIIS0140-6736(20)31094-1.pdf. Published 2020. Accessed June 9, 2020.

8. Hennon T, Penque M, Abdul-Aziz R et al. COVID-19 associated Multisystem Inflammatory Syndrome in Children (MIS-C) guidelines; a Western New York approach. Prog Pediatr Cardiol . 2020;57:101232. doi:10.1016/j.ppedcard.2020.101232

9. Rcpch.ac.uk. https://www.rcpch.ac.uk/sites/default/files/2020-05/COVID-19-Paediatricmultisystem-\%20inflammatory\%20syndrome-20200501.pdf. Published 2020. Accessed June 9, 2020.

\begin{tabular}{ll}
\hline Abbreviations & Abbreviations \\
\hline MIS-C & Multi-System Inflammatory Syndrome in Children \\
KD & Kawasaki Disease \\
\hline
\end{tabular}

